\title{
A Comparative Study on Linear Regression-Based Noise Estimation for Hyperspectral Imagery
}

\author{
Lianru Gao, Member, IEEE, Qian Du, Senior Member, IEEE, Bing Zhang, Senior Member, IEEE, Wei Yang, and \\ Yuanfeng $\mathrm{Wu}$
}

\begin{abstract}
In the traditional signal model, signal is assumed to be deterministic, and noise is assumed to be random, additive and uncorrelated to the signal component. A hyperspectral image has high spatial and spectral correlation, and a pixel can be well predicted using its spatial and/or spectral neighbors; any prediction error can be considered from noise. Using this concept, several algorithms have been developed for noise estimation for hyperspectral images. However, these algorithms have not been rigorously analyzed with a unified scheme. In this paper, we conduct a comparative study for such linear regression-based algorithms using simulated images with different signal-to-noise ratio (SNR) and real images with different land cover types. Based on experimental results, instructive guidance is concluded for their practical applications.
\end{abstract}

Index Terms-Hyperspectral, multiple linear regressions, noise estimation.

\section{INTRODUCTION}

$\mathbf{T}$ HE development of high spectral resolution image sensors improves the capability of monitoring the Earth system and human activity on the Earth. Due to the fact that hyperspectral imaging spectrometers adopt very narrow band intervals, energy acquired in each band is not enough to generate high signal-to-noise ratio (SNR). Spectral features in hyperspectral imagery (HSI) can be easily confused as a result of noise influence. Only when the level of noise is quantitatively lower than the depth of spectral absorption, the spectral feature can be recognized [1]. In HSI processing, the performance of many algorithms commonly is affected by noise, such as feature extraction [2], classification [3], spectral unmixing [4], and target detection [5], because most of these algorithms assume a signal/noise model in their formulas. Hence, accurate noise estimation can be beneficial for them in HSI information exploitation.

The noise in HSI usually belongs to one of two types: random noise and periodic noise. Periodic noise has fixed pattern and

Manuscript received July 09, 2012; revised August 14, 2012 and September 19, 2012; accepted September 24, 2012. Date of publication January 17, 2013; date of current version May 13, 2013. This work was supported by National Key Technology R\&D Program of China under Grant 2011BAH12B04, and by National Natural Science Foundation of China under Grant 40901225 and Grant 41201356 (Corresponding author: B. Zhang).

L. R. Gao, B. Zhang, W. Yang, and Y. F. Wu are with the Institute of Remote Sensing and Digital Earth, Chinese Academy of Sciences, Beijing 100094, China (e-mail: lrgao@ceode.ac.cn; zhangbing@ceode.ac.cn; wyang@ceode.ac.cn; yfwu@ceode.ac.cn).

Q. Du is with the Department of Electrical and Computer Engineering, Mississippi State University, Starkville, MS 39762 USA (e-mail: du@ece.msstate. edu).

Color versions of one or more of the figures in this paper are available online at http://ieeexplore.ieee.org.

Digital Object Identifier 10.1109/JSTARS.2012.2227245 can be removed using suitable procedures. However, random noise cannot be predicted in advance and also cannot be removed completely [6]-[8]. Therefore, random noise mainly is concerned in information exploitation. The random noise in HSI is generally assumed to be additive and uncorrelated with the signal [6]-[11]. The signal model described here can be expressed as

$$
x_{i, j, k}=s_{i, j, k}+n_{i, j, k}
$$

where $x_{i, j, k}$ is the image digital number (DN) at coordinate $(i, j)$ in band $k, s_{i, j, k}$ and $n_{i, j, k}$ are signal and noise components of $x_{i, j, k}$, respectively. The noise in HSIs generally is a normally distributed, zero-mean random process with a Gaussian probability density function (PDF), $f(x)$, given as

$$
f(x)=\frac{1}{\sqrt{2 \pi} \sigma} e^{-x^{2} / 2 \sigma^{2}}
$$

where $\sigma$ is the standard deviation (SD) of the noise. Here, it is assumed that signal is deterministic and random noise is additive and uncorrelated to signal. Note that we focus on the estimation of noise standard deviation in each band.

A HSI has high spatial and spectral correlation, and a pixel can be well predicted using its spatial and/or spectral neighbors; any prediction error can be considered from noise [9]. Using this concept, several algorithms have been developed for hyperspectral noise estimation. One simple noise estimation algorithm, referred to as the homogeneous area (HA) method, uses the mean of standard deviations of several visually homogeneous regions as noise estimate [10]. However, in this method, the homogeneous areas within an image need to be manually selected, and in most cases, the homogeneous areas are not existent or difficult to be identified. Curran and Dungan [1] developed the geo-statistical (GS) method, where some narrow homogeneous strips are selected to estimate the noise. Like HA, this method is also difficult to be automated, and requires to predetermine strictly homogeneous areas. Another disadvantage of these two methods is that the noise estimated from local homogeneous segments can not represent the noise level of the entire image. To improve the performance, several algorithms are developed to more reliably estimate noise with less human intervention, such as local means and local standard deviations (LMLSD) method [11], spectral and spatial de-correlation (SSDC) method [9], homogeneous regions division and spectral de-correlation (HRDSDC) method [12], and residual-scaled local standard deviation (RLSD) method [13]. Up to now, these algorithms were partially compared using few HSIs only, and have not been rigorously analyzed under a unified scheme. In this paper, we investigate their performance when noise level and image con- 


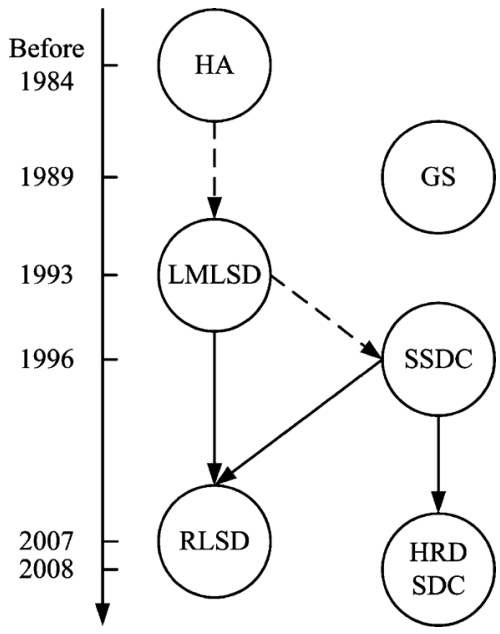

Fig. 1. Developing course of noise estimation algorithms, where real line presents improvement, and dashed line means that one algorithm is developed to deal with questions in another algorithm.

tents are changed. The result and conclusion will be beneficial for appropriate algorithm selection in image pre-processing.

The remainder of this paper is organized as follows. Section II focuses on an overview of several linear regression-based noise estimation algorithms, such as LMLSD, SSDC, HRDSDC, and RLSD. Section III describes experimental image database, accuracy assessment framework, and comparative analysis methods. In Section IV, comparative performance analysis for these algorithms using simulated and real hyperspectral images are presented and discussed. Section V draws the conclusions.

\section{LineAR REgRESSION-BASED NOISE ESTIMATION ALGRITHMS}

Hyperspectral data are corrupted by wavelength-dependent and sensor-specific noise, which significantly impacts data and resulting data products. Modeling this noise is an important topic related to hyperspectral sensing [14], [15]. During past years, several algorithms have been developed to estimate noise in HSIs, as shown in Fig. 1. HA is one of the earliest noise estimation algorithms, and still is used in laboratory or for hyperspectal image only acquired at calibration site. GS is seldomly used or improved in applications. LMLSD is developed to solve some questions shown in HA, such as low applicability and automaticity. To reduce low sensitivity with land cover types shown in LMLSD, SSDC is developed. RLSD and HRDSDC can be considered as improvements of LMLSD and SSDC. In this paper, only noise estimation algorithms based on signal independent model are analyzed and assessed. There are also some new algorithms with signal dependent model lately [16], [17]. These algorithms are not discussed in this paper.

\section{A. Description of Main Algorithms}

1) LMLSD: The LMLSD algorithm assumed that an HSI is made up of many homogeneous blocks and few heterogeneous blocks. Statistical analysis of these blocks can be used to estimate noise. In LMLSD, an entire image is uniformly divided into non-overlapping small blocks with $w \times w$ pixels. The local mean (LM) of each block in band $k$ is calculated by

$$
L M=\frac{1}{w^{2}} \sum_{i=1}^{w} \sum_{j=1}^{w} x_{i, j, k}
$$

Here, LM of a block is related to the signal, and it is assumed that the deviation from the local mean is due to noise. Thus, the local standard deviation (LSD) of each block is estimated by

$$
L S D=\left[\frac{1}{\left(w^{2}-1\right)} \sum_{i=1}^{w} \sum_{j=1}^{w}\left(x_{i, j, k}-L M\right)^{2}\right]^{\frac{1}{2}}
$$

where LSD is considered as noise standard deviation of the block. Within the range of the minimum and maximum value of these standard deviations, a number of bins of equal width are delineated. The number of blocks falling into each bin is counted, and the bin with the largest number of blocks represents the noise standard deviation of the entire image. The mean value of LSDs in this bin is considered as the estimated noise of the HSI.

2) SSDC: In SSDC, the high between-band (spectral) and within-band (spatial) correlations are used to de-correlate an image data via linear regression, and the remaining unexplained residuals are the estimates of noise. In this method, the image also is uniformly divided into non-overlapping small blocks with $w \times w$ pixels. The pixel value at $(i, j)$ in band $k$ can be predicted by its spatial and spectral neighbors and the residual $r_{i, j, k}$ is calculated as

$$
r_{i, j, k}=x_{i, j, k}-\hat{x}_{i, j, k}
$$

where $\hat{x}_{i, j, k}$ is the linear predicted value of $x_{i, j, k}$, and is computed as

$$
\hat{x}_{i, j, k}=a+b x_{i, j, k-1}+c x_{i, j, k+1}+d x_{p, k}
$$

where $p$ expresses spatial adjacent pixel of $x_{i, j, k}, a, b, c$, and $d$ are the coefficients determined by multiple linear regressions (MLR). The LSD of all the residuals in a block is computed using

$$
L S D=\left[\frac{1}{w^{2}-4} \sum_{i=1}^{w} \sum_{j=1}^{w} r_{i, j, k}^{2}\right]^{\frac{1}{2}}
$$

The mean value of these standard deviations is considered as the noise estimate for the entire image. Note that (5) and (6) use two spectral neighbors and one spatial neighbor for MLR. If more neighbors of $x_{i, j, k}$ are also used, then more coefficients are to be determined, similarly with the MLR method.

3) HRDSDC: In most remote sensing images, the scene consists primarily of many regions arranged in a patchwork manner, where each region corresponds to one class of land cover type. These homogeneous regions are usually related to objects in the scene. When the sampling interval is smaller than the size of an earth object, the probability of transition from state $i$ to state $j$ is much greater if $j=i$, than if $j \neq i$. Based on this theory, the segmentation algorithm can be used to divide an image into 
TABLE I

Summary of Noise Estimation Algorithms Discussed In This PAPER

\begin{tabular}{|c|c|c|c|c|}
\hline Algorithm & Nature & Hypotheses & Key procedure \\
\hline HA & Supervised & Image contains homogeneous area in each object & ROI selection, LSD calculation \\
\hline GS & Supervised & Image contains homogeneous area in each object & ROI selection, semi-variance calculation, curve fitting & Spatial \\
\hline LMLSD & $\begin{array}{c}\text { Near- } \\
\text { automated }\end{array}$ & Image mainly contains homogeneous blocks & Block partition, LSD statistics \\
\hline RLSD & $\begin{array}{c}\text { Near- } \\
\text { automated }\end{array}$ & High spectral correlation & Spatial & Spatial \\
\hline SSDC & $\begin{array}{c}\text { Near- } \\
\text { automated }\end{array}$ & High spectral and spatial correlations & BLR, LSD statistics \\
\hline HRDSDC & $\begin{array}{c}\text { Near- } \\
\text { automated }\end{array}$ & Object internal regularity, high spectral correlation & Spatial \\
Spectral
\end{tabular}

many homogeneous regions, and to produce a class map of the scene. In this class map, each region contains one earth object, and each spectrum of the same region has a similar correlation between the data in one band and the data in its two adjacent bands. HRDSDC employs this general internal regularity of earth objects in natural scene and the strong spectral correlations of HSIs. In HRDSDC, an image segmentation algorithm is applied first to partition the image into spectrally homogeneous regions. Then, MLR is applied to each homogeneous region to calculate residuals using spectral neighbors as follows

$r_{i, j, k}=x_{i, j, k}-\hat{x}_{i, j, k}$, where $\hat{x}_{i, j, k}=a+b x_{i, j, k-1}+c x_{i, j, k+1}$

where $a, b$, and $c$ are the coefficients computed using MLR. At last, the mean value of residual standard deviations in local regions is used as the global noise estimate.

4) RLSD: RLSD can be considered an effective improvement of LMLSD. In each block, instead of using LSD as local noise estimate, the residuals from the MLR in the spectral domain are used for local noise estimation. Therefore, RLSD also incorporates the merit of SSDC. Following LMLSD and SSDC, RLSD also adopts the strategy to divide a band image into many small blocks. Then the residuals in each block are estimated by using MLR as shown in (8). This procedure leaves residuals which are much more likely noise than the original data used in LMLSD. The standard deviation value of residuals, so-called LSD of residuals, actually approaches the noise in the block. RLSD adopts the same statistical methods described by Gao [11] to obtain the best estimate of band noise. The histogram of these LSDs is computed, and the one with the maximum counts is treated as the noise standard deviation for the entire image. In general, RLSD can produce more accurate estimate than LMLSD.

\section{B. Summary}

A brief overview of noise estimation algorithms investigated in this paper is summarized in Table I. It can be seen that all of them are developed based on some assumptions. For examples, HA and GS assume that land cover of an image is strictly homogeneous, So they are suitable to images containing homogeneous area which is covered by only one earth object. LMLSD assumes that an image mainly contains many homogeneous blocks, and is suitable to images with relatively high spatial resolution and simple texture. On the contrary, RLSD, SSDC and HRDSDC assume high spectral correlation, which is reasonable for HSIs.

Although these noise estimation algorithms make different assumptions, they have similar three steps, as shown in Fig. 2: 1) image spatial partition, 2) LSD estimation of noise, and 3) global noise estimation based on local information. In HA and GS, to get strictly homogeneous area, region of interest (ROI) selection is the key procedure; LMLSD, RLSD, and SSDC divide an image into many blocks, and the block size selection is a factor influencing the performance; in HRDSDC, image segmentation is used for spatial partition. When image spatial partition is finished, LSD estimation of noise will be applied on these blocks or homogeneous regions. LMLSD uses original data to calculate LSD; on the contrary, RLSD, SSDC, and HRDSDC use residuals calculated by linear regression to estimate noise. As for global noise estimation, two methods are adopted: LSD statistics method by LMLSD and RLSD, and the mean value of residual LSDs by SSDC and HRDSDC.

\section{EXPERIMENTAL DESIGN}

This section designs two experiments to assess performances of noise estimation algorithms. The first experiment based on simulated images is to validate the accuracy of noise estimates for images with different values of SNR. The second experiment based on real images is to assess the robustness of noise estimation algorithms for images with land cover change.

\section{A. Comparison With Simulated Images}

To generate simulated images, the linear mixture model (LMM) is used with specific level of additive noise. Let $\mathbf{x}(i, j)$ be a pixel at spatial coordinate $(i, j)$. It can be considered as a $K$-dimensional ( $K$-D) vector, where $K$ is the number of spectral bands. This spectrum may be modeled in terms of a linear combination of several endmembers using the following expression [4]:

$\mathbf{x}(i, j)=\sum_{m=1}^{M} c_{m} \mathbf{e}_{m}=\mathbf{E c}$, where $\sum_{m=1}^{M} c_{m}=1,0 \leq c_{m} \leq 1$

where $M$ is the number of endmembers, and $c_{m}$ is a scalar value representing the fractional coverage of endmember vector $e_{m}$ 
HA

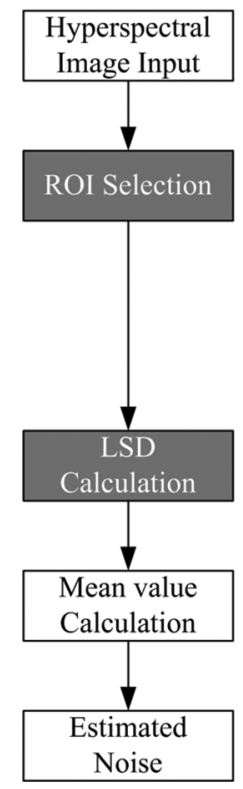

GS

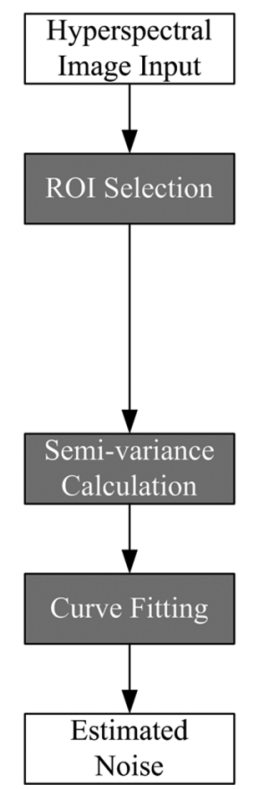

LMLSD

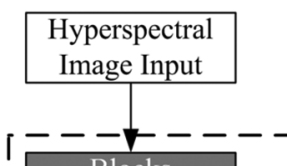

RLSD

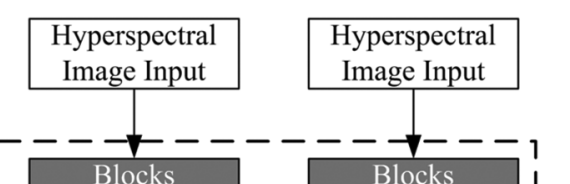

HRDSDC

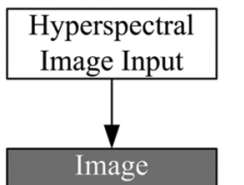

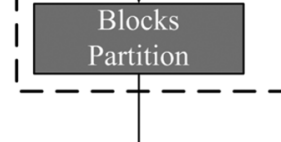

Partition

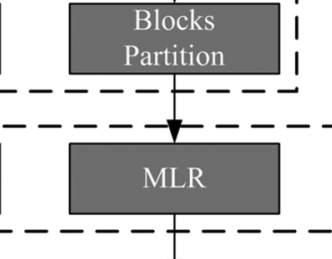

Segmentation

Fig. 2. Summary of primary techniques used in noise estimation algorithms.
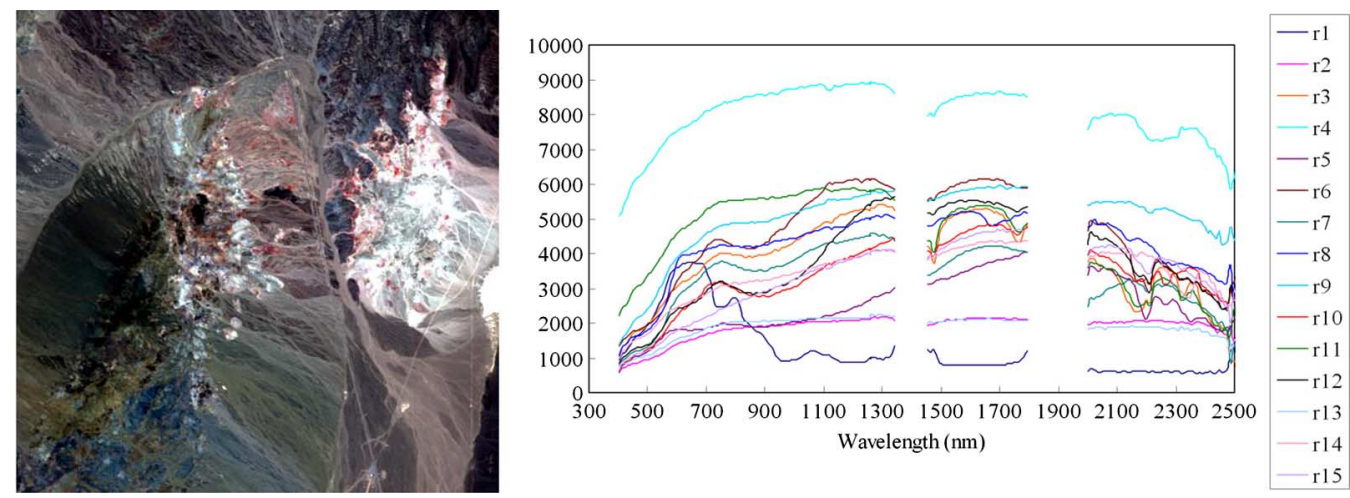

Fig. 3. AVIRIS image of Cuprite (left) and endmember spectra (right) extracted by NFINDR.

in pixel $\mathbf{x}(i, j)$. With additive noise [4], [18], [19], (9) can be rewritten as:

$$
\begin{aligned}
\mathbf{x}(i, j) & =\sum_{m=1}^{M} c_{m} \mathbf{e}_{m}+\mathbf{n}(i, j)=\mathbf{E} \mathbf{c}+\mathbf{n}=\mathbf{s}+\mathbf{n} \\
\text { where } \sum_{m=1}^{M} c_{m} & =1,0 \leq c_{m} \leq 1
\end{aligned}
$$

where $\mathbf{n}$ can be modeled by using classical additive white Gaussian noise [20].

As shown in Fig. 3, a Cuprite image collected by airborne visible/infrared imaging spectrometer (AVIRIS) on 19/06/1997 is used to generate a simulated image with controlled noise level, which consists of $500 \times 500$ pixels and 221 spectral bandswith a nominal ground resolution of $20 \mathrm{~m}$, spectral resolution of $10 \mathrm{~nm}$, and 16 bit radiometric resolution. This image is a typical data for spectral unmixing research and application. Many spectral unmixing algorithms based on LMM have been successfully tested through Cuprite image shown in Fig. 3
[21]-[25]. In this study, endmember spectra and abundance maps are extracted with minimum noise fraction (MNF) transform [26], N-FINDR method [21], and unconstrained least squares method [18]. N-FINDR is one of the most widely used algorithms for endmember extraction. However, due to the requirement of volume calculation algorithm used in N-FINDR, a first step of dimensionality reduction should be performed by using MNF transform [21], [27]. Then endmember spectra and abundance maps are combined to form a new HSI using (9). The detail flow of this experiment design is shown in Fig. 4. Due to the controlled environment, true noise level is known for precise assessment of noise estimates. Here, two simple statistical measurements are used to evaluate the error of estimated noise: mean of absolute error (MAE) and standard deviation of absolute error (SDAE), which can be represented as

$$
\operatorname{MAE}\left(n_{k}, \hat{n}_{k}\right)=\frac{1}{K} \sum_{k=1}^{K}\left|n_{k}-\hat{n}_{k}\right|
$$




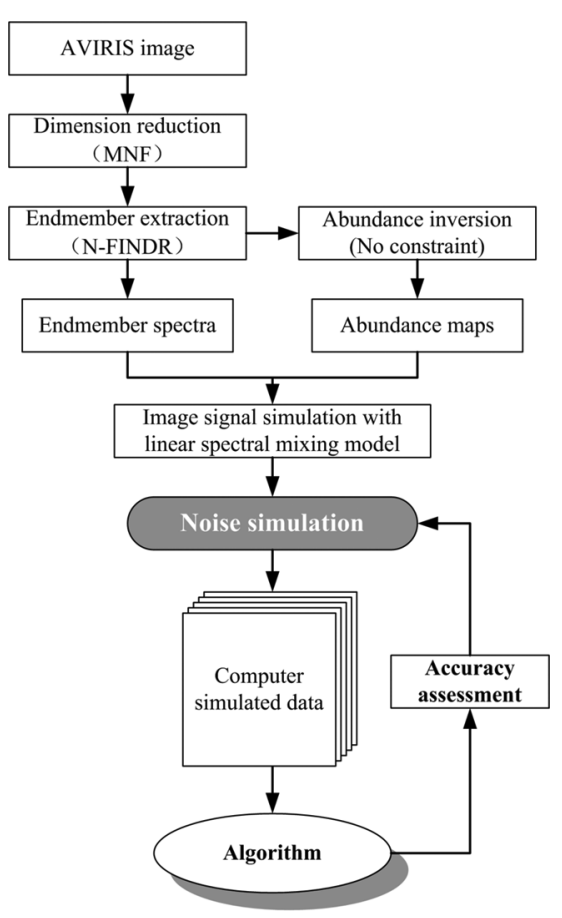

Fig. 4. Schematical description of the approach used to assess noise estimation accuracy with simulated data.

where $n_{k}$ and $\hat{n}_{k}$ are true and estimated noise value at band $k$, respectively, and

$$
\mathrm{SDAE}=\left(\frac{1}{K} \sum_{k=1}^{K}\left(\left|n_{k}-\hat{n}_{k}\right|-\mathrm{MAE}\right)^{2}\right)^{\frac{1}{2}}
$$

In addition, for statistical experiment used in this part, we also provide significance level analysis of noise estimation results based on T-test.

In this study, based on estimation of the virtual dimensionality, 14 components of MNF are selected for N-FINDR to extract 15 endmembers. Reflectance spectra of these endmembers are shown in Fig. 3. Note that, in this study, precision of endmember extraction and abundance inversion is not important; instead, combination result of endmember spectra and abundance maps is more concerned. 15 abundance maps and one error map of Cuprite image shown in Fig. 3 are calculated by unconstrained least squares method using radiance spectra of these endmembers. In image simulation, the error map considered as uncertain information is ignored. Therefore, these radiance spectra of endmembers and abundance maps are combined to generate a new image. The simulated image and its kaolinite spectrum are shown in Fig. 5. It can be seen that spatial texture and spectral curve of simulated image are similar to the original image. Due to the fact that these endmember spectra and abundance maps are treated as signals, the simulated image is considered as combination of signal. Thus, different levels of Gaussian random noise are added, which is determined by SNR, such as 150:1, 110:1, 90:1, 70:1, 50:1, 30:1, and 10:1. In this simulation experiment, SNR is specified as the ratio between signal level and the SD of the simulated noise [28]. Simulated image and
TABLE II

DetAiled Description of AVIRIS IMAges SHOWN IN Fig. 1

\begin{tabular}{|c|c|c|c|c|}
\hline Fig. 7 & $\begin{array}{c}\text { Spatial } \\
\text { Resolution }\end{array}$ & Acquired site & $\begin{array}{c}\text { Acquired } \\
\text { time }\end{array}$ & Image description \\
\hline (a) & $20 \mathrm{~m}$ & Cuprite & 19/06/1997 & $\begin{array}{c}\text { Homogeneous } \\
\text { minerals }\end{array}$ \\
\hline (b) & $20 \mathrm{~m}$ & Lunar Lake & $23 / 06 / 1997$ & Homogeneous gob \\
\hline (c) & \multirow[b]{2}{*}{$20 \mathrm{~m}$} & \multirow[b]{2}{*}{ Jasper Ridge } & \multirow[b]{2}{*}{ 03/04/1997 } & Heterogeneous city \\
\hline (d) & & & & $\begin{array}{c}\text { Homogeneous } \\
\text { vegetation }\end{array}$ \\
\hline (e) & \multirow[b]{2}{*}{$3.4 \mathrm{~m}$} & \multirow[b]{2}{*}{ Low Altitude } & \multirow[b]{2}{*}{ 05/07/1996 } & Heterogeneous city \\
\hline (f) & & & & $\begin{array}{l}\text { Homogeneous } \\
\text { farmland }\end{array}$ \\
\hline$(\mathrm{g})$ & \multirow[b]{2}{*}{$20 \mathrm{~m}$} & \multirow[b]{2}{*}{ Moffett Field } & \multirow[b]{2}{*}{ 20/06/1997 } & Heterogeneous city \\
\hline (h) & & & & $\begin{array}{c}\text { Homogeneous } \\
\text { water }\end{array}$ \\
\hline
\end{tabular}

kaolinite spectrum with SNR $=10: 1$ are shown in Fig. 6. It can be seen that spatial feature and spectral curve are degraded.

\section{B. Comparison With Real Images}

To assess the performance of aforementioned algorithms when land cover type is different, 8 real AVIRIS radiance images in Fig. 7 with very different land cover types are used in the experiment. Normally, the random noise in AVIRIS images is mainly additive and uncorrelated with the signal [17]. More detailed descriptions are shown in Table II. Note that the AVIRIS sensor is made up of four grating spectrometers, and detector read-out error is existent in band 33, so the noise estimation for this band is unreliable [29].

The AVIRIS HSIs in Fig. 7 were acquired from 07/1996 to 06/1997. During this time period, the sensor parameters were changed little and noise level may be similar in these images. In particular, Fig.7(c) and 7(d), Fig. 7(e) and 7(f), Fig. 7(g) and 7(h) are cut from the same image, respectively. Therefore, their noise level should be the same.

\section{RESULtS}

\section{A. Parameters Selection}

As summarized in Table I and Fig. 2, SSDC and HRDSDC adopt the mean value of LSDs of small blocks or local regions after MLR. On the contrary, in LMLSD and RLSD, noise is estimated through the statistical analysis of LSD histogram, so we use the parameters in [11] to find appropriate bins. In LMLSD and RLSD, bins are set in the range between the minimum LSD of all blocks and 1.2 times the average LSD of all blocks, and 150 bins are used for noise estimation.

The block size is the most important parameter for LMLSD, SSDC, and RLSD. In the experiment, the blocks of size $4 \times$ $4,5 \times 5,6 \times 6,7 \times 7$, and $8 \times 8$ are tested. For LMLSD, since it is assumed that an image mainly is composed of many homogeneous small blocks, $4 \times 4$ is an appropriate choice in our experiment. It seems that a smaller block size is also beneficial for SSDC and RLSD. However, when the block size is $4 \times 4$ or $5 \times 5$, some homogeneous blocks in most experimental data have similar DN value in certain bands; as a consequence, it 

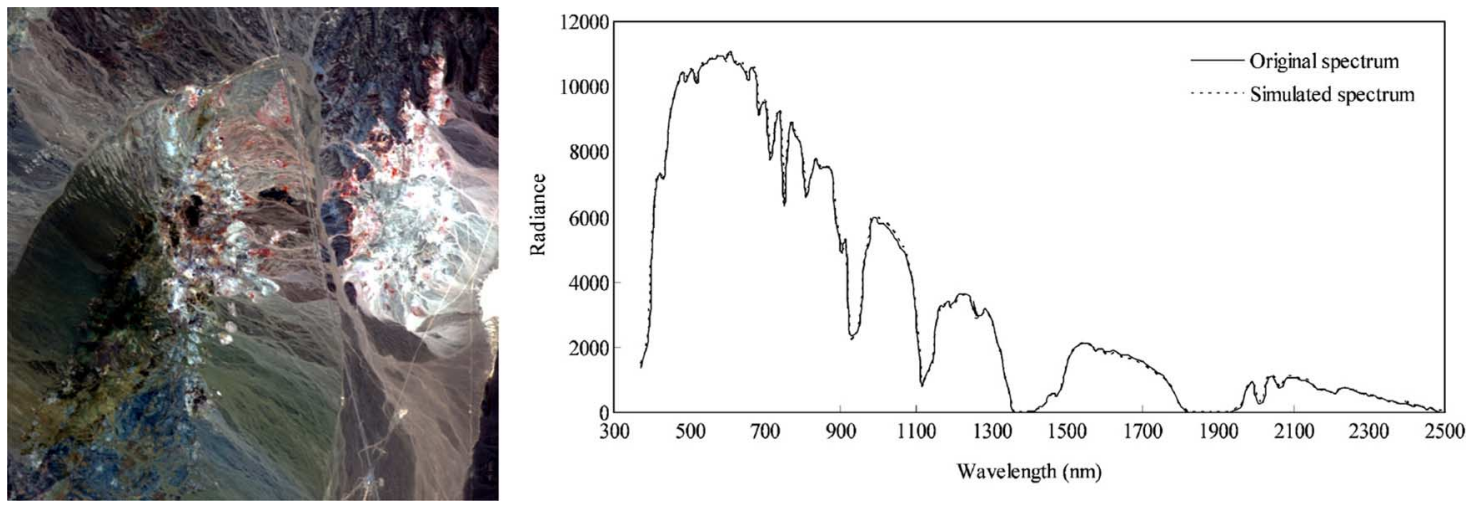

Fig. 5. Simulated image (left) and kaolinite spectrum (right) without noise.
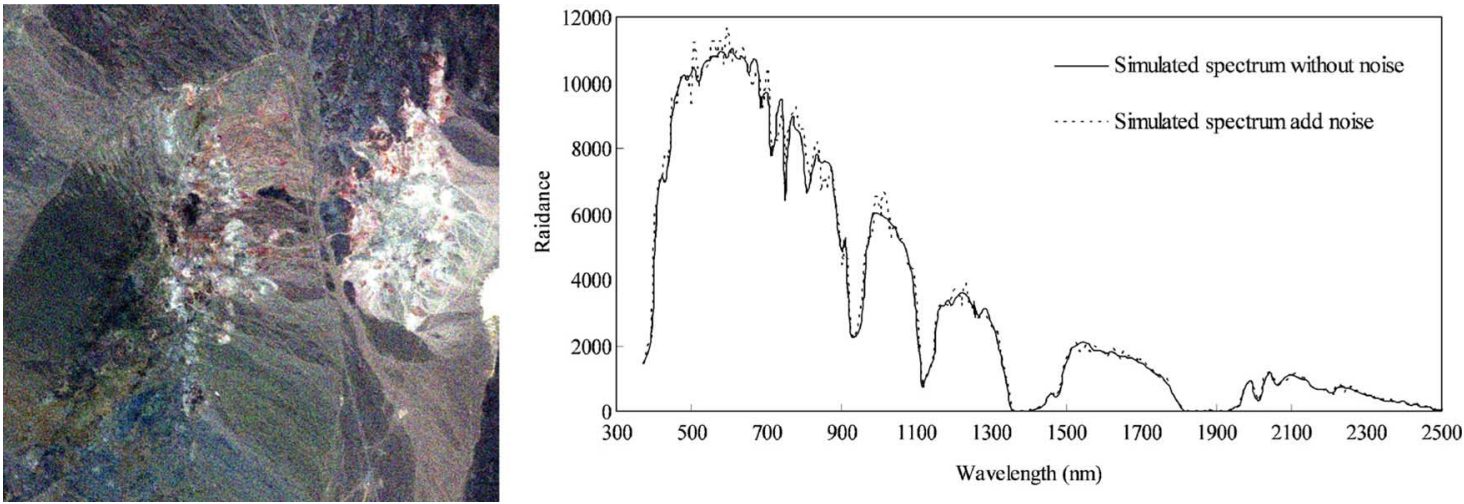

Fig. 6. Simulated image (left) and kaolinite spectrum (right) with SNR $=10: 1$ using signal independent noise model.

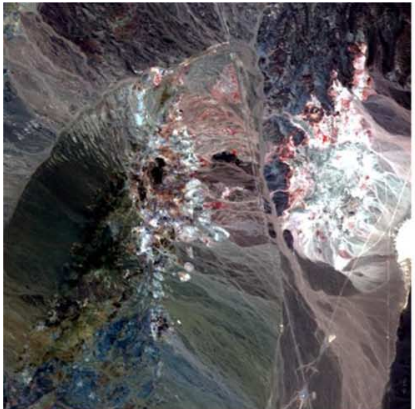

(a)

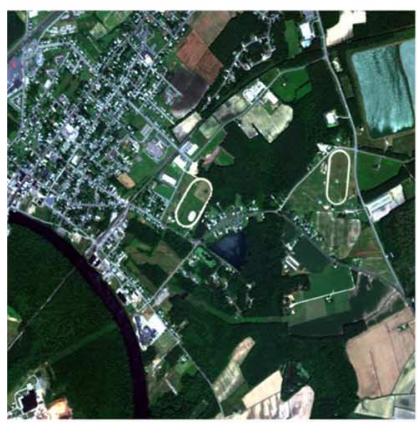

(e)

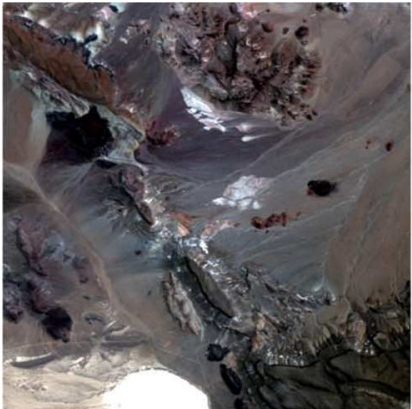

(b)

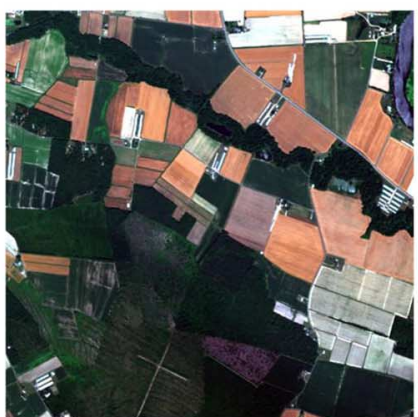

(f)

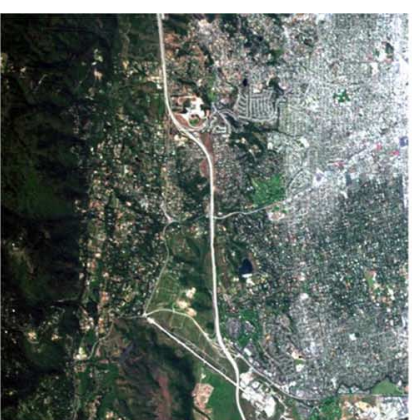

(c)

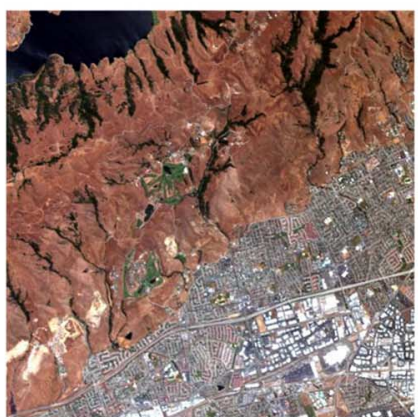

(g)

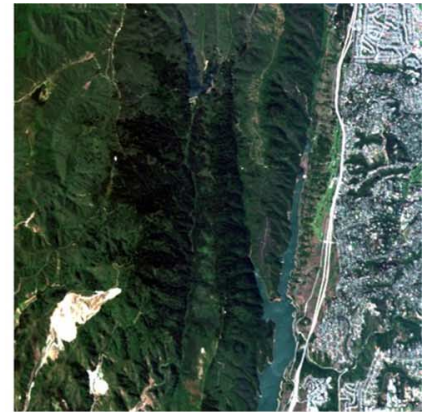

(d)

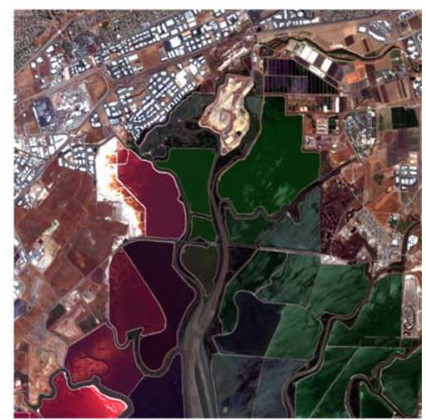

(h)

Fig. 7. AVIRIS radiance images used for noise estimation.

makes the inverse matrix calculation in MLR infeasible. Thus, the block of size $6 \times 6$ is adopted for SSDC and RLSD. As for the HRDSDC, it employs more advanced image segmentation, whose step and related parameters are the same as in [12].

\section{B. Results From Simulated Images}

Noise estimation results using LMLSD, SSDC, HRDSDC and RLSD are shown in Fig. 8(a)to Fig. 8(c) and Table III. It can be seen that: 
TABLE III

ACCURACy AsSESSment of Noise Estimation Algorithm Using SimUlated Imagery With Different SNR

\begin{tabular}{|c|c|c|c|c|c|c|c|c|c|c|c|c|c|c|c|c|}
\hline \multirow{3}{*}{ Method } & \multicolumn{16}{|c|}{ SNR/Simulated noise SD } \\
\hline & \multicolumn{2}{|c|}{$150: 1 / 12.75$} & \multicolumn{2}{|c|}{ 130:1/14.71 } & \multicolumn{2}{|c|}{ 110:1/17.39 } & \multicolumn{2}{|c|}{$90: 1 / 21.25$} & \multicolumn{2}{|c|}{$70: 1 / 27.33$} & \multicolumn{2}{|c|}{$50: 1 / 38.26$} & \multicolumn{2}{|c|}{$30: 1 / 63.76$} & \multicolumn{2}{|c|}{$10: 1 / 191.28$} \\
\hline & $\mathrm{MAE}$ & SDAE & MAE & \begin{tabular}{|l} 
SDAE \\
\end{tabular} & MAE & \begin{tabular}{|l} 
SDAE \\
\end{tabular} & $\mathrm{MAE}$ & SDAE & MAE & SDAE & MAE & SDAE & MAE & SDAE & MAE & SDAE \\
\hline & & & & & & & 14 & & & & 11 & & & & 88 & 12.64 \\
\hline & & & & & & & & & & & & 7 & & & & 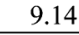 \\
\hline nes & & & 4. & 4. & & 4. & 5. & & & & 9.2 & 8.69 & 14.61 & 14.02 & 37.94 & 37.9 \\
\hline RLSD & 2.98 & 3.18 & 3.25 & 3.46 & 3.82 & 3.84 & 4.29 & \begin{tabular}{l|l}
4.31 \\
\end{tabular} & 5.19 & 5.17 & 6.68 & 6.67 & 9.03 & 9.72 & 9.33 & 12.0 \\
\hline
\end{tabular}

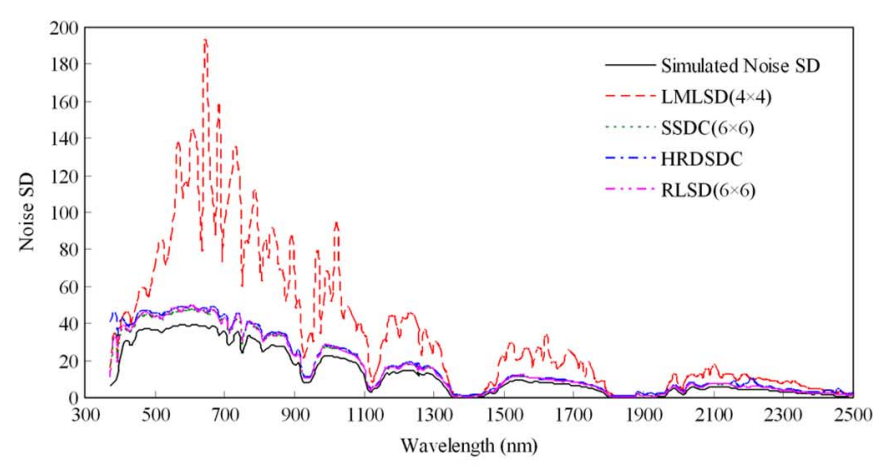

(a)

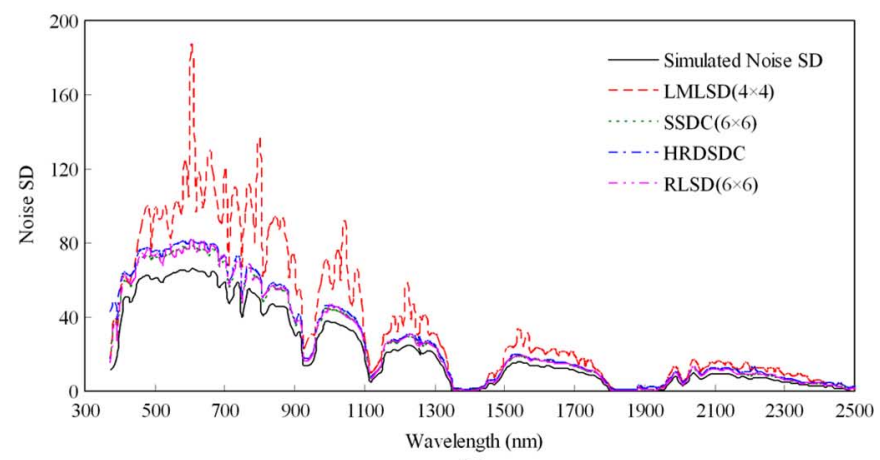

(b)

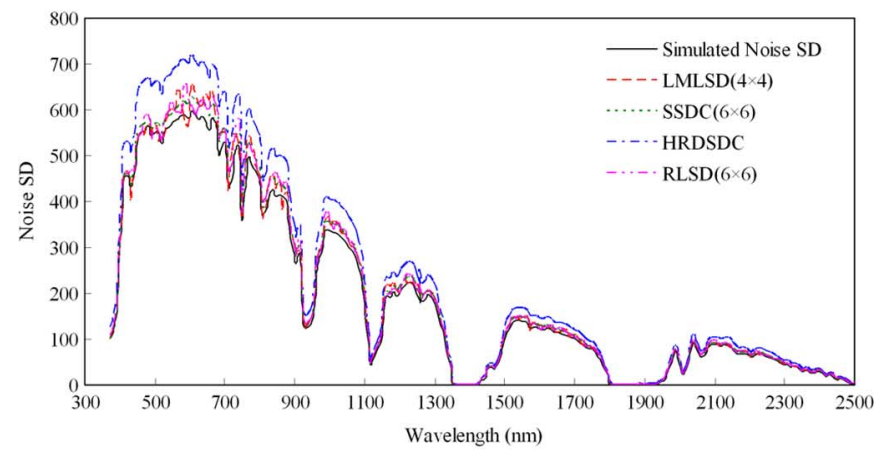

(c)

Fig. 8. Noise estimation results for simulated HSIs, where SNR of (a) is 150:1, (b) is $90: 1$, and (c) is $10: 1$.

1) SSDC is the most reliable algorithm for noise estimation of HSIs with different levels of SNR. The capability of RLSD and HRDSDC are also good enough in this experiment. However, LMLSD can be easily affected by land cover types. Therefore, it is not satisfied for most images with different level of SNR.

2) In most cases, estimated noise using SSDC, HRDSDC, and RLSD is a little greater than true noise value. It is because that inner variability between bands (resulting from input of endmember spectra) and intra-pixel variability (resulting from spatially heterogeneous pixel contents) are considered as noise when MLR are used in these methods. The absolute error between estimation result and simulated noise is increasing with increase of noise level. On the contrary, the relative error defined as the ratio of MAE/SDAE to simulated noise $\mathrm{SD}$ is decreasing with increase of noise level.

3) To LMLSD, the estimation result grows better in precision with increase of noise level. When the SNR of image is not very low, estimated noise is largely different from truth noise value. If SNR of image is decreased to 10:1, estimated noise is nearly the same as truth noise value.

4) Due to noise impact, error of image segmentation used in HRDSDC is increasing with increase of noise level. When SNR is greater than 50:1, estimated noise is satisfied. However, when SNR is very low, such as 30:1 or 10:1, estimated noise using HRSDC is the worst among all the noise estimation algorithms.

Significance levels of noise estimation results are analyzed by T-test, and analysis results are shown in Table IV. When $\mathrm{P} \gg 0.05$ significance level, it means that the estimated noise has no statistic difference from the true value. It can be seen that:

1) With the SNR value being decreased, the $P$ value is increased.

2) For various SNR values, SSDC performs the best, and RLSD is the second best.

3) When SNR > 50:1, HRDSDC performs better than LMLSD. However, due to influence of error in image segmentation, when SNR $<50: 1$, the estimates from HRDSDC still have significant difference from the true values.

4) When SNR > 50:1, LMLSD should not be used.

\section{Results From Real Images}

1) Noise Estimation With Different Land Cover Types: Fig. 9(a) shows the noise estimates from LMLSD for the 8 real HSI scenes in Fig. 7. They are varied along with land cover types, which is not reasonable. It may be because that most image blocks do not meet the homogeneous hypothesis made by LMLSD, in particular, when an image is simply uniformly partitioned. Only when the primary land cover type is water, the image can be homogeneous enough to find adequately effective blocks. Experimental results show that noise estimates from SSDC (Fig. 9(b)) is better than LMLSD. HRDSDC, considered as modified SSDC using image segmentation as spatial partition, generated also better result as shown in Fig. 9(c). Noise estimates shown in Fig. 9(d) using RLSD are also better in terms that noise estimates are less sensitive to image contents. 


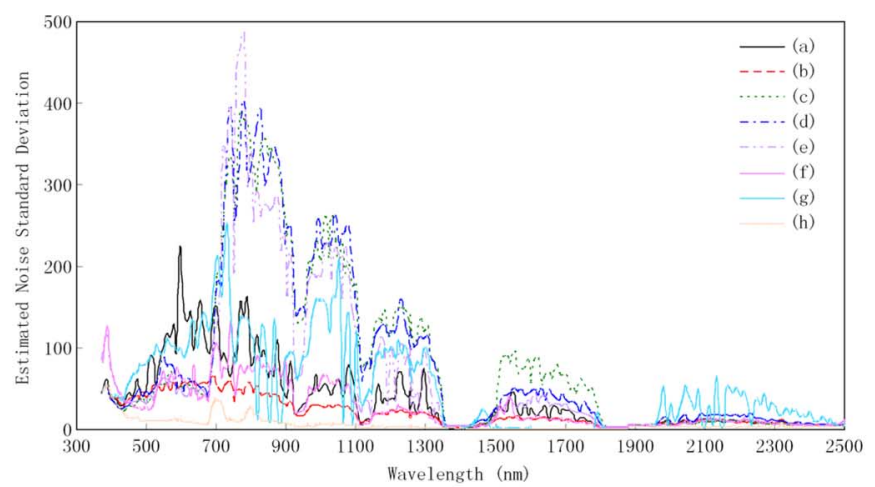

(a)

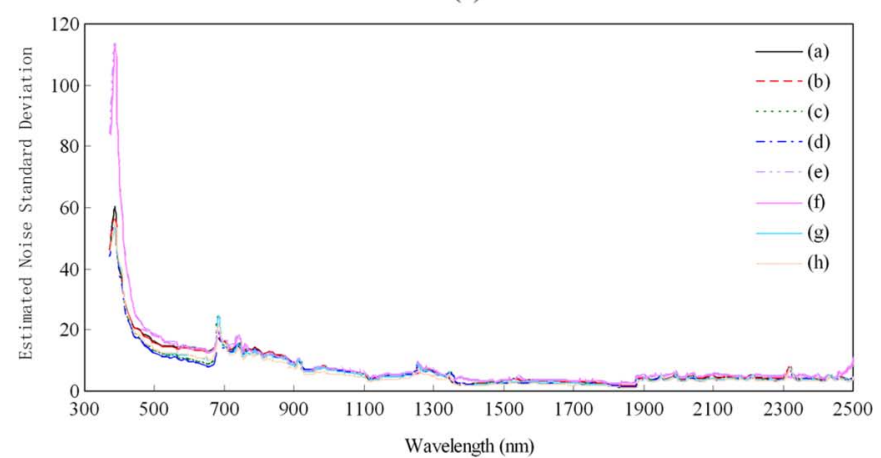

(b)

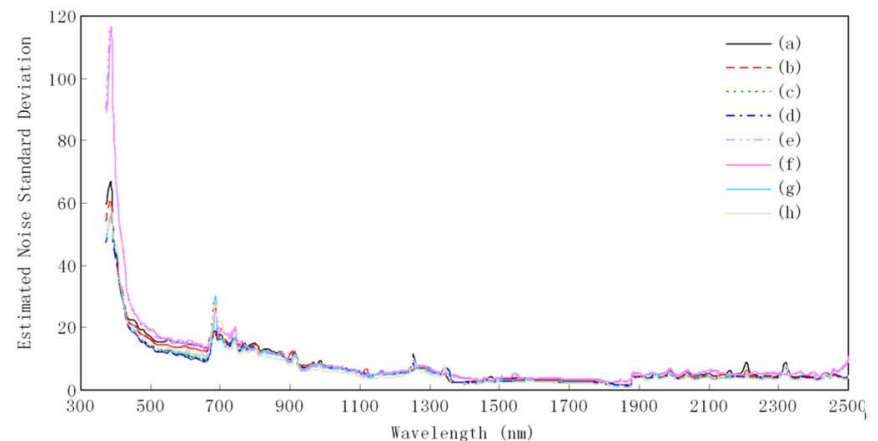

(c)

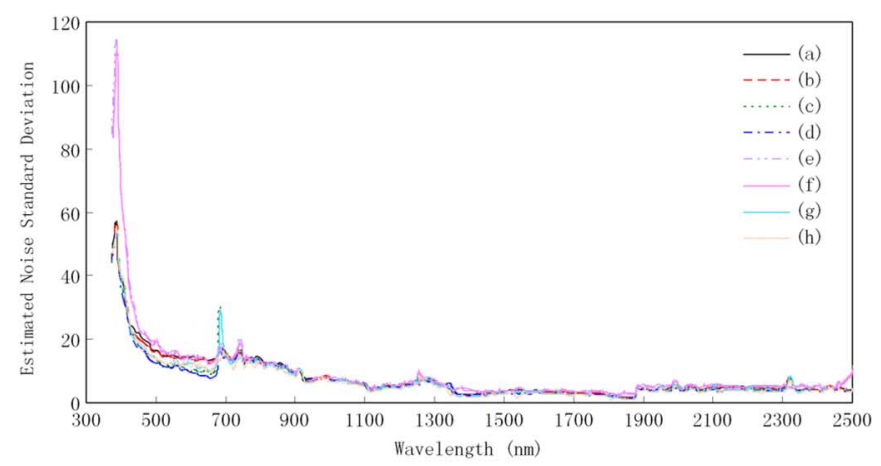

(d)

Fig. 9. Noise estimation results of Fig. 7 using (a) LMLSD, (b) SSDC, (c) HRDSDC, and (d) RLSD.

Though these algorithms consider spectral and spatial information in different ways, the noise estimated results shown in Fig. 10 are very similar. To quantitatively compare the stability of these algorithms for different land cover types, MAE and SDAE expressed in (11) and (12) are used to analyze error of noise estimation results. Here, through results shown in Fig. 9, it can be seen that results of LMLSD are not correct, and results of

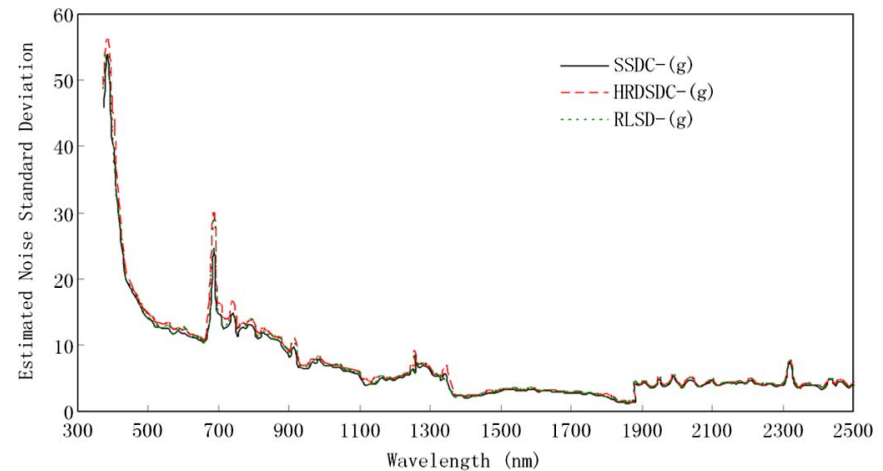

Fig. 10. Noise curves in estimated noise image of Fig. 7(g) using SSDC, HRDSDC, and RLSD.

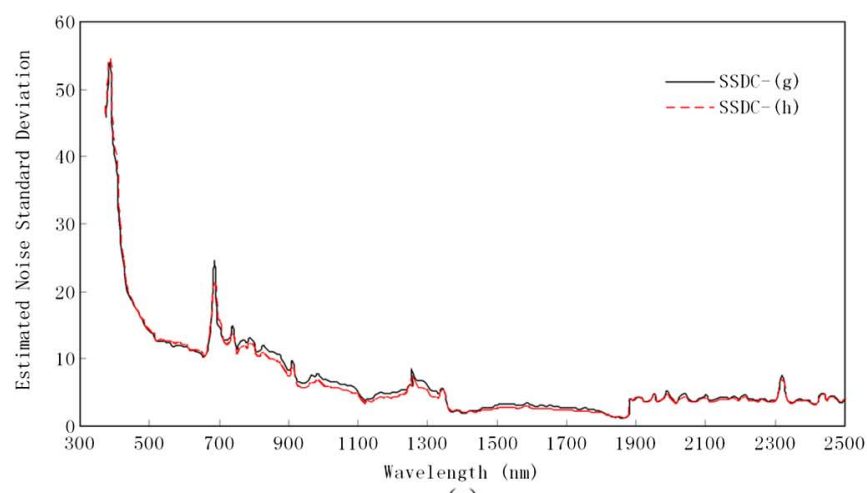

(a)

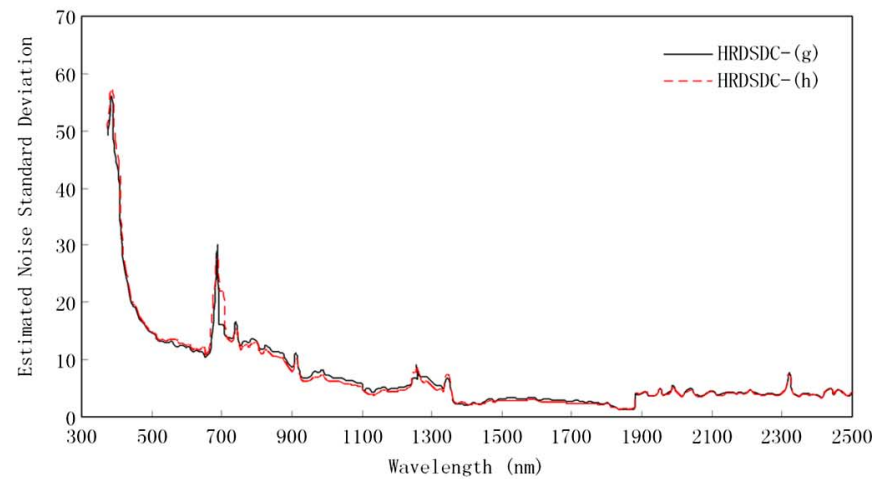

(b)

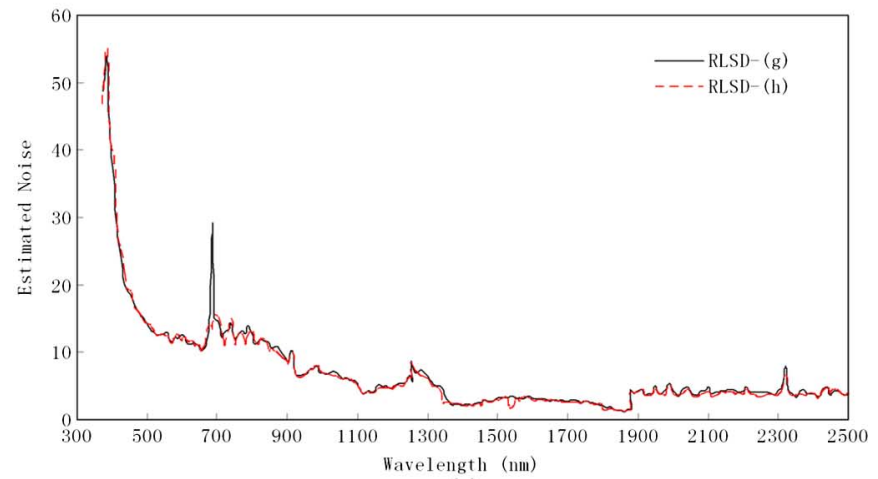

(c)

Fig. 11. Noise estimation results of Fig. 7(g) and (h) using (a) SSDC, (b) HRDSDC, and (c) RLSD. These two subimages are cropped from a single image, but Fig. 7(h) is mainly covered by water.

SSDC, HRDSDC, and RLSD are similar and stable for very different land cover types. Therefore, in this study, the mean value 
TABLE IV

SignificANCE ANALYSis OF NOISE Estimation RESUlts

\begin{tabular}{|c|r|r|r|r|r|r|r|r|}
\hline \multirow{2}{*}{ Method } & \multicolumn{7}{|c|}{ SNR/Simulated noise SD } \\
\cline { 2 - 9 } & $150: 1 / 12.75$ & $130: 1 / 14.71$ & $110: 1 / 17.39$ & $90: 1 / 21.25$ & $70: 1 / 27.33$ & $50: 1 / 38.26$ & $30: 1 / 63.76$ & $10: 1 / 191.28$ \\
\hline LMLSD & $1.91 \times 10^{-15}$ & $1.51 \times 10^{-13}$ & $4.76 \times 10^{-11}$ & $1.011 \times 10^{-8}$ & $4.10 \times 10^{-6}$ & $6.86 \times 10^{-4}$ & 0.0246 & 0.6079 \\
\hline SSDC & $6.58 \times 10^{-3}$ & $8.64 \times 10^{-3}$ & 0.0114 & 0.0155 & 0.0228 & 0.0384 & 0.0918 & 0.5212 \\
\hline HRDSDC & $1.85 \times 10^{-4}$ & $3.70 \times 10^{-4}$ & $7.03 \times 10^{-4}$ & $1.27 \times 10^{-3}$ & $2.19 \times 10^{-3}$ & $3.54 \times 10^{-3}$ & $5.55 \times 10^{-3}$ & 0.0144 \\
\hline RLSD & $4.65 \times 10^{-3}$ & $6.85 \times 10^{-3}$ & $6.94 \times 10^{-3}$ & 0.0121 & 0.0175 & 0.0269 & 0.0666 & 0.4984 \\
\hline
\end{tabular}

TABLE V

Accuracy Assessments of Noise Estimation Algorithm Using ReAl IMAgery With Different Land Cover Types

\begin{tabular}{|c|c|c|c|c|c|c|c|c|c|c|c|c|c|c|c|c|}
\hline \multirow{3}{*}{ Method } & \multicolumn{16}{|c|}{ Real AVIRIS HSIs with very different land cover types } \\
\hline & \multicolumn{2}{|c|}{ (a) } & \multicolumn{2}{|c|}{ (b) } & \multicolumn{2}{|c|}{ (c) } & \multicolumn{2}{|c|}{ (d) } & \multicolumn{2}{|c|}{ (e) } & \multicolumn{2}{|c|}{ (f) } & \multicolumn{2}{|c|}{ (g) } & \multicolumn{2}{|c|}{ (h) } \\
\hline & MAE & SDAE & MAE & SDAE & MAE & SDAE & MAE & SDAE & MAE & SDAE & MAE & SDAE & MAE & SDAE & MAE & SDAE \\
\hline LMLSD & 28.64 & 37.06 & 12.55 & 13.02 & 73.66 & 96.35 & 71.59 & 96.70 & 56.07 & 93.39 & 17.61 & 22.20 & 44.25 & 50.41 & 1.42 & 2.57 \\
\hline SSDC & & & & 0.24 & 0.13 & 0.15 & 0.14 & 0.19 & 0.17 & 0.24 & 0.14 & 0.20 & 0.15 & 0.21 & 0.25 & 0.31 \\
\hline HRDSDC & 4 & & 0 & 0.53 & 0.24 & 0.37 & 0.27 & .43 & 0.29 & 0.48 & 0.29 & 0.52 & 0.22 & 0.40 & 0.30 & 0.66 \\
\hline RLSD & 0.24 & 0.48 & 0.17 & 0.34 & 0.16 & 0.28 & 0.17 & 0.30 & 0.20 & 0.33 & 0.21 & 0.36 & 0.15 & 0.28 & 0.28 & 0.42 \\
\hline
\end{tabular}

TABLE VI

AcCuracy Assessments of Noise Estimation Algorithm Using Real IMAGERY WITH DIFFERENT LAND COVER TYPES

\begin{tabular}{|c|r|r|r|r|r|r|}
\hline \multirow{3}{*}{ Method } & \multicolumn{6}{|c|}{ Pairs of test image } \\
\cline { 2 - 7 } & \multicolumn{1}{|c|}{ Fig. 7(c) and (d) } & Fig. 7(e) and (f) & Fig. 7(g) and (h) \\
\cline { 2 - 7 } & \multicolumn{1}{|c|}{ MAE } & \multicolumn{1}{c|}{ SDAE } & MAE & SDAE & MAE & SDAE \\
\hline LMLSD & 12.83 & 14.73 & 40.58 & 75.05 & 44.42 & 50.00 \\
\hline SSDC & 0.21 & 0.32 & 0.14 & 0.17 & 0.48 & 0.33 \\
\hline HRDSDC & 0.18 & 0.21 & 0.12 & 0.20 & 0.54 & 0.61 \\
\hline RLSD & 0.35 & 0.43 & 0.32 & 0.40 & 0.41 & 0.42 \\
\hline
\end{tabular}

of noise estimates of SSDC, HRDSDC, and RLSD for one certain land cover type is treated as the reliable noise value of this land cover type, namely $\hat{n}_{k}$; corresponding to this mean value, MAE and SDAE of LMLSD, SSDC, HRDSDC, and RLSD are calculated for each land cover type as shown in Table V. One can see that results of LMLSD are far different from others. Results of LMLSD highly depend on homogeneity of land cover type; for examples, results of homogeneous gobi and farmland shown in Fig. 7(b) and (f) are better than results of heterogeneous city, and result of homogeneous water shown in Fig. 7(g) is the best. From results shown in Table V, it also can be seen that stability of SSDC for different land cover types is a little better than those of other methods.

2) Noise Estimation for Images Covered by Water: Areas covered by water always are very homogeneous. However, spectrum of water from near infrared to shortwave infrared spectroscopy is nearly equal to zero. Therefore, many methods may yield lower noise estimate than the actual value (e.g., results shown in Fig. 11(a) by SSDC and Fig. 11(b) by HRDSDC). This is because that their procedure of mean value calculation of LSDs cannot avoid the influence of very low radiance, and is not suitable for noise estimation of images mainly covered by water. However, RLSD produced better results as shown in Fig. 11(c), which may be because that it uses the LSD with the maximum occurrence as the noise estimate for the image.

In this study, a quantitative analysis of noise estimation for water area is also carried out based on MAE and SDAE. Here, two images in one pair are treated as $n_{k}$ and $\hat{n}_{k}$ respectively. Results are shown in Table VI. Although SSDC and HRDSDC are reliable for images covered by city and vegetation, noise estimation results of image covered mainly by water is not satisfied. On the contrary, RLSD expresses good performance on images mainly covered by water, such as Fig. 7(h).

\section{CONCLUSION}

Over the past years, several linear regression-based noise estimation algorithms have been developed for HSIs containing uncorrelated random noise. However, these algorithms have not been rigorously analyzed with a unified scheme. In this paper, we conduct a comparative study for these algorithms using simulated images with different SNR and real images with different land cover types. Specially, based on image simulation, we design a new framework to assess and compare the quantitative performance of noise estimation algorithms. Parameters of these algorithms are also discussed and well confirmed.

Experiments with simulated and real HSIs indicate that 1) using the residual of linear regression is an effective approach for noise estimation; and 2) when the noise level is not very low, the combination of spectral and spatial information in linear regression provides better result than the algorithms using spatial information only. It also can be concluded that: 1) The simple uniform spatial partition with small block size, say, $6 \times 6$, can perform as well as more sophisticated image segmentation; and 2) the histogram of LSD is more appropriate than the simple average of LSD for global noise estimation.

\section{ACKNOWLEDGMENT}

The authors acknowledge the Jet Propulsion Laboratory for generously providing the AVIRIS data. The authors also acknowledge the two anonymous reviewers for their outstanding comments and suggestions, which greatly helped to improve the technical content and presentation of the manuscript.

\section{REFERENCES}

[1] P. J. Curran and J. L. Dungan, "Estimation of signal-to-noise: A new procedure applied to AVIRIS data," IEEE Trans. Geosci. Remote Sens., vol. 27, no. 5, pp. 620-628, Sep. 1989. 
[2] Q. Du, N. Raksuntorn, S. Cai, and R. J. Moorhead, II, "Color display for hyperspectral imagery," IEEE Trans. Geosci. Remote Sens., vol. 46, no. 6 , pp. $1858-1866$, Jun. 2008.

[3] G. Camps-Valls, L. Gómez-Chova, J. Calpe-Maravilla, J. D. MartínGuerrero, E. Soria-Olivas, L. Alonso-Chordá, and J. Moreno, "Robust support vector method for hyperspectral data classification and knowledge discovery," IEEE Trans. Geosci. Remote Sens., vol. 42, no. 7, pp. 1530-1542, Jul. 2004.

[4] A. Plaza, P. Martínez, R. Pérez, and J. Plaza, "A quantitative and comparative analysis of endmember extraction algorithms from hyperspectral data," IEEE Trans. Geosci. Remote Sens., vol. 42, no. 3, pp. 650-663, Mar. 2004.

[5] D. W. J. Stein, S. G. Beaven, L. E. Hoff, E. M. Winter, A. P. Schaum, and A. D. Stocker, "Anomaly detetction from hyperspectral imagery," IEEE Signal Process. Mag., vol. 19, no. 1, pp. 58-69, Jan. 2002.

[6] D. A. Landgrebe and E. Malaret, "Noise in remote-sensing systems: The effect on classification error," IEEE Trans. Geosci. Remote Sens., vol. 24, pp. 294-299, Mar. 1986.

[7] G. M. Smith and P. J. Curran, "Methods for estimating image signal-tonoise ratio," in Advances in Remote Sensing and GIS Analysis, P. M. Atkinson and N. J. Tate, Eds., 1st ed. : Wiley, 1999, vol. 5, pp. 61-74.

[8] B. R. Corner, R. M. Narayanan, and S. E. Reichenbach, "Noise estimation in remote sensing imagery using data masking," Int. J. Remote Sen., vol. 24, no. 4, pp. 689-702, 2003.

[9] R. E. Roger and J. F. Arnold, "Reliably estimating the noise in AVIRIS hyperspectral images," Int. J. Remote Sen., vol. 17, no. 10, pp. 1951-1962, 1996.

[10] R. C. Wrigley, D. H. Card, C. A. Hlavka, J. R. Hall, F. C. Mertzz, C. Archwamety, and R. A. Schowengerdt, "Thematic Mapper image quality: Registration, noise, and resolution," IEEE Trans. Geosci. Remote Sens., vol. GE-22, no. 3, pp. 263-271, May 1984.

[11] B. C. Gao, "An operational method for estimating signal to noise ratios from data acquired with imaging spectrometers," Remote Sens. Environ., vol. 43, no. 1, pp. 23-33, 1993.

[12] L. R. Gao, B. Zhang, X. Zhang, W. J. Zhang, and Q. X. Tong, "A new operational method for estimating noise in hyperspectral images," IEEE Geosci. Remote Sens. Lett., vol. 5, no. 1, pp. 83-87, Jan. 2008.

[13] L. R. Gao, B. Zhang, J. T. Wen, and Q. Ran, "Residual-scaled local standard deviations method for estimating noise in hyperspectral images," in Proc. SPIE, Wuhan, China, 2007, vol. 6787, p. 678713-1.

[14] Q. Du, "Noise estimation for remote sensing image data analysis," in Proc. SPIE, Bellingham, WA, 2003, vol. 5159, pp. 246-254.

[15] J. Chanussot, M. M. Crawford, and B.-C. Kuo, "Foreword to the special issue on hyperspectral image and signal processing," IEEE Trans. Geosci. Remote Sens., vol. 48, no. 11, pp. 3871-3876, Nov. 2010.

[16] M. L. Uss, B. Vozel, V. V. Lukin, and K. Chehdi, "Local signal-dependent noise variance estimation from hyperspectral textural images," IEEE J. Sel. Top. Signal Process., vol. 5, no. 3, pp. 469-486, Jun. 2011.

[17] N. Acito, M. Diani, and G. Corsini, "Signal-dependent noise modeling and model parameter estimation in hyperspectral images," IEEE Trans. Geosci. Remote Sens., vol. 49, no. 8, pp. 2957-2971, Aug. 2011.

[18] N. Keshava and J. F. Mustard, "Spectral unmixing," IEEE Signal Process. Mag., vol. 19, no. 1, pp. 44-57, Jan. 2002.

[19] J. M. Bioucas-Dias, A. Plaza, N. Dobigeon, M. Parente, Q. Du, P. Gader, and J. Chanussot, "Hyperspectral unmixing overview: Geometrical, statistical, and sparse regression-based approaches," IEEE J. Sel. Top. Signal Process., vol. 5, no. 2, pp. 354-379, Apr. 2012.

[20] O. Eches, N. Dobigeon, and J. Y. Tourneret, "Enhancing hyperspectral image unmixing with spatial correlations," IEEE Trans. Geosci. Remote Sens., vol. 49, no. 11, pp. 4239-4247, Nov. 2011.

[21] M. E. Winter, "N-FINDR: An algorithm for fast autonomous spectral end-member determination in hyperspectral data," in Proc. SPIE, Denver, CO, 1999, vol. 3753, pp. 266-275.

[22] J. M. P. Nascimento and J. M. B. Dias, "Vertex component analysis: A fast algorithm to unmix hyperspectral data," IEEE Trans. Geosci. Remote Sens., vol. 43, no. 4, pp. 898-910, Apr. 2005.

[23] H. L. Li and L. P. Zhang, "A hybrid automatic endmember extraction algorithm based on a local window," IEEE Trans. Geosci. Remote Sens., vol. 49, no. 11, pp. 4223-4238, Nov. 2011.

[24] B. Somers, M. Zortea, A. Plaza, and G. P. Asner, "Automated extraction of image-based endmember bundles for improved spectral unmixing," IEEE J. Sel. Topics Appl. Earth Observ. Remote Sens. (JSTARS), vol. 5, no. 2, pp. 396-408, Apr. 2012.

[25] G. Martin and A. Plaza, "Spatial-spectral preprocessing prior to endmember identification and unmixing of remotely sensed hyperspectral data," IEEE J. Sel. Topics Appl. Earth Observ. Remote Sens. (JSTARS), vol. 5, no. 2, pp. 380-395, Apr. 2012.
[26] A. A. Green, M. Berman, P. Switzer, and M. D. Craig, "A transformation for ordering multispectral data in terms of image quality with implications for noise removal," IEEE Trans. Geosci. Remote Sens., vol. 26, no. 1, pp. 65-74, Jan. 1988.

[27] J. W. Boardman and F. A. Kruse, "Analysis of imaging spectrometer data using N-dimensional geometry and a mixture-tuned matched filtering approach," IEEE Trans. Geosci. Remote Sens., vol. 49, no. 11, pp. 4138-4152, Nov. 2011.

[28] K. Segl, L. Guanter, C. Rogass, T. Kuester, S. Roessner, H. Kaufmann, B. Sang, V. Mogulsky, and S. Hofer, "EeteS-The EnMAP end-to-end simulation tool," IEEE J. Sel. Topics Appl. Earth Observ. Remote Sens. (JSTARS), vol. 5, no. 2, pp. 522-530, Apr. 2012.

[29] R. O. Green, M. L. Eastwood, C. M. Sarture, T. G. Chrien, M. Aronsson, B. J. Chippendale, J. A. Faust, B. E. Pavri, C. J. Chovit, M. Solis, M. R. Olah, and O. Williams, "Imaging spectroscopy and the airborne visible/infrared imaging spectrometer (AVIRIS)," Remote Sens. Environ., vol. 65, no. 3, pp. 227-248, 1998.

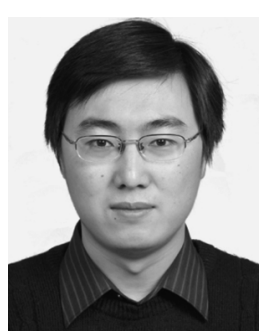

Lianru Gao (M'12) received the B.S. degree in civil engineering from Tsinghua University, Beijing, China, in 2002 and the Ph.D. degree in cartography and geographic information system from Institute of Remote Sensing Applications, Chinese Academy of Sciences, Beijing, China, in 2007.

$\mathrm{He}$ is currently an Associate Professor with the Institute of Remote Sensing and Digital Earth, Chinese Academy of Sciences. He has published over thirty papers in China and abroad. His research interests include spectral feature analysis, hyperspectral image processing, target detection, and image simulation.

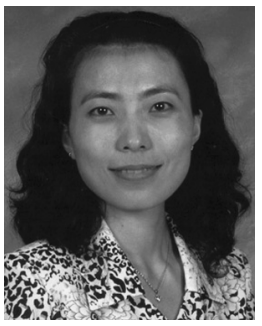

Qian Du (S'98-M'00-SM'05) received her Ph.D. degree in electrical engineering from University of Maryland Baltimore County in 2000.

She was with the Department of Electrical Engineering and Computer Science, Texas A\&M University-Kingsville, from 2000-2004. She joined the Department of Electrical and Computer Engineering at Mississippi State University in Fall 2004, where she is currently an Associate Professor. Her research interests include hyperspectral remote sensing image analysis, pattern classification, data compression, and

neural networks.

Dr. Du currently serves as Co-Chair for the Data Fusion Technical Committee of IEEE Geoscience and Remote Sensing Society. She also serves as Associate Editor for IEEE Journal of Selected Topics in Applied Earth Observations and Remote Sensing and Associate Editor for IEEE SIGNAL PROCESSING LETTERS. She received the 2010 Best Reviewer award from IEEE Geoscience and Remote Sensing Society. Dr. Du is the General Chair for the 4th IEEE GRSS Workshop on Hyperspectral Image and Signal Processing: Evolution in Remote Sensing (WHISPERS) in Shanghai, China in 2012. Dr. Du is a member of SPIE, ASPRS, and ASEE.

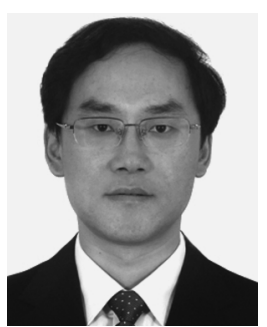

Bing Zhang (M'11-SM'12) received the B.S. degree in geography from Peking University, Shenzhen, China, the MS and Ph.D. degrees in remote sensing from the Institute of Remote Sensing Applications, Chinese Academy of Sciences (CAS), Beijing, China.

Currently, he is a Professor and the Deputy Director of the Institute of Remote Sensing and Digital Earth, CAS. He specializes in Hyperspectral Remote Sensing and has more than 17 years of experience in studying and graduate education in this field. His study interests include development of physics-based models and image processing software for the use of hyperspectral remote sensing data in solving problems in geology, hydrology, ecology and botany. It is remarkable that some innovative research and research-based development projects undertaken by him that have made him received several Chinese National, Ministerial and Provincial S\&T progress awards. 


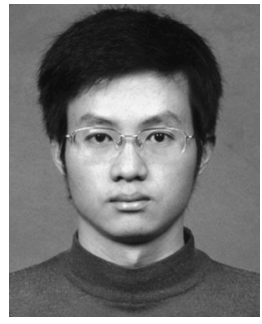

Wei Yang received the B.S. degree in electronic engineering from Harbin Institute of Technology, China, in 2010. He also received the B.A. degree in economics from the same university in 2010 . He is now studying toward the M.S. degree in electronic engineering at the Institute of Remote Sensing and Digital Earth, Chinese Academy of Sciences.

His current research interests include hyperspectral image processing, artificial neural network and real-time signal processing. He is a member of Sigma $\mathrm{Xi}$.

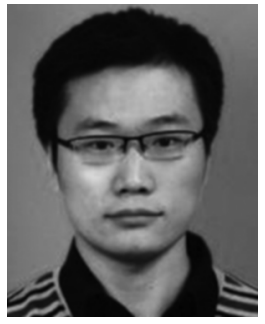

Yuanfeng $\mathrm{Wu}$ received the B.S., M.S. degrees in computer science from the China University of Mining and Technology, Beijing, China in 2004 and 2007 and the Ph.D. degree in cartography and geographical information system from the Graduate University of Chinese Academy of Sciences, Beijing, China, in 2010.

$\mathrm{He}$ is currently an Assistant Professor in the Institute of Remote Sensing and Digital Earth, Chinese Academy of Sciences. His main research interests include the high performance computing algorithms in hyperspectral image processing and computer software. 